\title{
Total Synthesis of Lycogarubin C and Lycogalic Acid
}

\author{
James S. Oakdale and Dale L. Boger ${ }^{*}$ \\ Department of Chemistry and The Skaggs Institute for Chemical Biology, The Scripps Research \\ Institute, 10550 North Torrey Pines Road, La Jolla, California 92037
}

\section{Abstract}

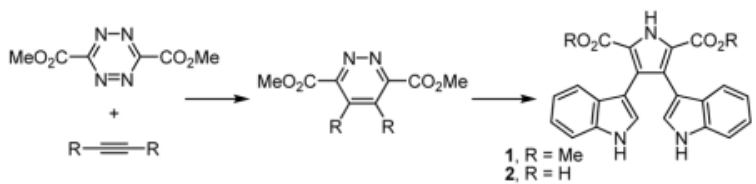

Two complementary concise total syntheses of lycogarubin C (1) and lycogalic acid (2, aka chromopyrrolic acid) are detailed utilizing a 1,2,4,5-tetrazine $\rightarrow$ 1,2-diazine $\rightarrow$ pyrrole Diels-Alder strategy and enlisting acetylenic dienophiles.

\begin{abstract}
Lycogarubin C (1) and lycogalic acid (2) were first identified as natural products in 1994, having been isolated independently by Steglich ${ }^{1}$ and Akazawa ${ }^{2}$ from Lycogala epidendrum, a slime mold (Figure 1). More recently, lycogalic acid, also referred to as chromopyrrolic acid (CPA) ${ }^{3}$ has been identified as a common intermediate in the biosynthesis of the indolo[2,3a]carbazole alkaloids including rebeccamycin (6) and staurosporine (7) that exhibit broad spectrum activity as inhibitors of protein kinases as well as Topoisomerase I. ${ }^{4}$ As the efforts to elucidate the details of this biosynthetic pathway have progressed, the oxidation of chromopyrrolic acid (2) to 4 via 3 has attracted considerable interest since it involves an unusual oxidative aryl-aryl coupling reaction. ${ }^{3,5}$ Moreover, in exploration of the individual enzymecatalyzed steps in the pathway, $\mathbf{5}$ was isolated as an aerobic product following the oxidative coupling of 2 effected by RebP/StaP. ${ }^{6}$ As an off pathway intermediate that does not lead to formation of $\mathbf{4}$, it is likely that 5 and related compounds may well constitute the newest members of this class of natural products. As a result, we initiated efforts on the synthesis of $\mathbf{1}$ and $\mathbf{2}$ that in turn may serve as synthetic as well biosynthetic precursors to these potential newest members of this class of natural products.
\end{abstract}

Complementary to reports of the synthesis of $\mathbf{1}$ or $\mathbf{2}^{1,7-9}$ we anticipated that $\mathbf{1}$ and $\mathbf{2}$ would be readily accessible through use of a 1,2,4,5-tetrazine $\rightarrow$ 1,2-diazine $\rightarrow$ pyrrole Diels-Alder strategy that appears ideally suited for their preparation. ${ }^{10}$ Thus, the inverse electron demand Diels-Alder reaction of a 1,2-bis(indol-3-yl)acetylene (8) with dimethyl 1,2,4,5-tetrazine-3,6dicarboxylate (9) ${ }^{11}$ followed by a reductive ring contraction reaction of the resulting 1,2diazine $^{12}$ to a dimethyl pyrrole-2,5-dicarboxylate could directly provide 1 or a protected penultimate precursor (Figure 2). Moreover, the potential use of the mono methyl esters derived from such dimethyl pyrrole-2,5-dicarboxylates to directly access products like $\mathbf{5}$ via a unique oxidative decarboxylation reaction ${ }^{13}$ provided the additional incentive for us to pursue the synthesis of $\mathbf{1}$ and $\mathbf{2}$. The recent disclosure of Fu and Gribble ${ }^{9}$ reporting that this direct strategy was not successful and their development of a clever alternative, using an olefinic versus

boger@scripps.edu.

Supporting Information Available. Full experiment details and compound characterizations are provided. This material is available free of charge via the internet at http://pubs.acs.org. 
acetylenic dienophile, provided the incentive for us to disclose our related, but more successful observations utilizing acetylenic dienophiles.

The initial route explored entailed implementing the Diels-Alder reaction of the 1,2-bis-[ $[(\mathrm{N}$ methoxycarbonyl)-indol-3-yl]acetylene (8) with 1,2,4,5-tetrazine $\mathbf{9}$, Scheme 1 . The preparation of the indole substituted acetylene $\mathbf{8}$ began with iodination of indole followed by immediate methyl carbamate protection of the sensitive indole providing 10. Stepwise Sonogashira coupling of $\mathbf{1 0}$ first with trimethylsilylacetylene (82\%), TMS deprotection of $\mathbf{1 1}\left(\mathrm{Bu}_{4} \mathrm{NF}, \mathrm{THF}, 80 \%\right)$, and subsequent coupling of the resulting acetylene $\mathbf{1 2}$ again with $\mathbf{1 0}$ provided $\mathbf{8}(85 \%)$.

The Diels-Alder reaction of acetylene 8 with 9 provided $\mathbf{1 3}(65 \%)$ in a reaction that proved sluggish requiring $15 \mathrm{~d}$ in refluxing toluene $\left(110^{\circ} \mathrm{C}\right)$ with repetitive additions of the $1,2,4,5$ tetrazine 9 every $3 \mathrm{~d}$ as it slowly decomposes at this temperature. Use of higher reaction temperatures simply accelerated the decomposition of the 1,2,4,5-tetrazine 9 and did not lead to improvements in the rate or conversions to $\mathbf{1 3}$. Notably and although this result merits the examination of alternative approaches to the preparation of the 1,2-diazine 13, it was not as unsuccessful as reported by Fu and Gribble..$^{9}$ In fact, such 1,2- diarylacetylenes exhibit a reactivity that is dependent on the electronic character of the aryl groups. For example, although alkoxyphenyl substituents convey sufficient reactivity to such alkynes making their use synthetically attractive, $12 \mathrm{~d}, \mathrm{e}, \mathrm{g}$ the unsubstituted diphenylacetylene itself reacts with $\mathbf{9}$ only slowly. We found that $\mathbf{8}$ exhibits a reactivity that is slightly lower than that of diphenylacetylene, and that it not as reactive as a number of more productive acetylenic dienophiles.

The acetylene adopted for an alternative approach to $\mathbf{1 3}$ was 1,2-bis(tributylstannyl)acetylene (14). ${ }^{14}$ The reaction of $\mathbf{1 4}$ with dimethyl 1,2,4,5-tetrazine-3,6-dicarboxylate (9) proceeded smoothly in dioxane under mild thermal conditions $\left(45^{\circ} \mathrm{C}, 24 \mathrm{~h}\right)$ and provided the Diels-Alder product 15 in exceptional conversions (97\%). Subsequent Stille coupling of $\mathbf{1 0}$ with the resulting 1,2-diazine 15 proceeded effectively and twice providing the same key 4,5-bis (indol-3-yl)-1,2-diazine 13 in good yield (70\%). In the optimization of this reaction, $\left(\mathrm{Ph}_{3} \mathrm{P}\right)_{2} \mathrm{PdCl}_{2}$ proved more effective than $\left(\mathrm{Ph}_{3} \mathrm{P}\right)_{4} \mathrm{Pd}$, the addition of $\mathrm{CuI}$ or $\mathrm{CuCl}_{2}$ improved the initially modest conversions, and the additional inclusion of $\mathrm{LiCl}$ further improved the reaction eliminating a side reaction of proto deiodination.

Treatment of 13 with $\mathrm{Zn} / \mathrm{HOAc}$ (30 equiv $\mathrm{Zn}, \mathrm{HOAc}-\mathrm{CH}_{2} \mathrm{Cl}_{2} 1: 1,25^{\circ} \mathrm{C}, 12 \mathrm{~h}$ ) cleanly effected the reductive ring contraction reaction providing pyrrole $16(68 \%)$ and completing the 1,2,4,5tetrazine $\rightarrow 1$,2-diazine $\rightarrow$ pyrrole conversions originally envisioned. Selective removal of the indole $\mathrm{N}$-methoxylcarbonyl groups under mild conditions ( 2 equiv of $\mathrm{LiOH}, \mathrm{MeOH} / \mathrm{THF} /$ $\mathrm{H}_{2} \mathrm{O} 2: 2: 1,24^{\circ} \mathrm{C}, 12 \mathrm{~h}$ ) provided lycogarubin $\mathrm{C}(\mathbf{1})$ in good to excellent conversion (65-89\%), whereas exhaustive hydrolysis of $\mathbf{1 6}$ ( 7 equiv of $\mathrm{KOH}, \mathrm{THF} / \mathrm{H}_{2} \mathrm{O} 1: 1,45^{\circ} \mathrm{C}, 24 \mathrm{~h}$ ) or hydrolysis of 1 (3.5 equiv of $\mathrm{KOH}, \mathrm{THF} / \mathrm{H}_{2} \mathrm{O} 1: 1,45^{\circ} \mathrm{C}, 16 \mathrm{~h}$ ) afforded lycogalic acid (2) in superb conversion $(95 \%)$.

Thus, two complementary syntheses of $\mathbf{1}$ and $\mathbf{2}$ based on a 1,2,4,5-tetrazine $\rightarrow$ 1,2-diazine $\rightarrow$ pyrrole Diels-Alder strategy using acetylenic dienophiles are disclosed that extend our use of heterocyclic azadiene Diels-Alder reactions ${ }^{12,15}$ to a key biosynthetic precursor to the indolo $[2,3-a]$ carbazole alkaloids.

\section{Supplementary Material}

Refer to Web version on PubMed Central for supplementary material. 


\section{Acknowledgments}

We gratefully acknowledge the financial support of the National Institutes of Health (CA042056).

\section{References and Notes}

1. Frode R, Hinze C, Josten I, Schmidt B, Steffan B, Steglich W. Tetrahedron Lett 1994;35:1689.

2. Hashimoto T, Yasuda A, Akazawa K, Takaoka S, Tori M, Akazawa Y. Tetrahedron Lett 1994;35:2559.

3. (a) Hoshino T, Kojima Y, Hayashi T, Uchiyama T, Kaneko K. Biosci Biotech Biochem 1993;57:775. (b) Howard-Jones AR, Walsh CT. Biochemistry 2005;44:15652. [PubMed: 16313168] (c) HowardJones AR, Walsh CT. J Am Chem Soc 2006;128:12289. [PubMed: 16967980] (d) Nishizawa T, Gruschow S, Jayamaha DE, Nishizawa-Harada C, Sherman DH. J Am Chem Soc 2006;128:724. [PubMed: 16417354] (e) Asamizu S, Kato Y, Igarashi Y, Furumai T, Onaka H. Tetrahedron Lett 2006;47:473. (f) Sanchez CS, Brana AF, Mendez C, Salas JA. ChemBioChem 2006;7:1231. [PubMed: 16874749] (g) Chae C, Park J, Chung S, Kim T, Lee S, Yoon K, Shin J, Oh K. Bioorg Med Chem Lett 2009;19:1581. [PubMed: 19246195]

4. Review: Knolker H-J, Reddy KR. Chem Rev 2002;102:4303. [PubMed: 12428991]

5. (a) Makino M, Sugimoto H, Shiro Y, Asamizu S, Onaka H, Nagano S. Proc Natl Acad Sci USA 2007;104:11591. [PubMed: 17606921] (b) Wang Y, Chen H, Makino M, Shiro Y, Nagano S, Asamizu S, Onaka H, Shaik S. J Am Chem Soc 2009;131:6748. [PubMed: 19385626]

6. Howard-Jones AR, Walsh CT. J Am Chem Soc 2007;129:11016. [PubMed: 17705392]

7. Furstner A, Krause H, Thiel OR. Tetrahedron 2002;58:6373.

8. Hinze C, Kreipl A, Terpin A, Steglich W. Synthesis 2007:608.

9. Fu L, Gribble GW. Tetrahedron Lett 2010;51:537.

10. For reviews of heterocyclic azadiene cycloaddition reactions, see: (a) Boger DL. Tetrahedron 1983;39:2869. (b) Boger DL. Chem Rev 1986;86:781.(c) Boger, DL.; Weinreb, SM. Hetero DielsAlder Methodology in Organic Synthesis. Academic; San Diego: 1987.

11. (a) Boger DL, Panek JS, Coleman RS, Sauer J, Huber FX. J Org Chem 1985;50:5377. (b) Boger DL, Panek JS, Patel M. Org Synth 1992;70:79.

12. Review: (a) Joshi U, Pipelier M, Naud S, Dubreuil D. Curr Org Chem 2005;9:261.Applications: (b) Bach NJ, Kornfeld EC, Jones ND, Chaney MO, Dorman DE, Paschal JW, Clemens JA, Smalstig EB. J Med Chem 1980;23:481. [PubMed: 7189782] OMP: (c) Boger DL, Coleman RS, Panek JS, Yohannes D. J Org Chem 1984;49:4405.Prodigiosin: (d) Boger DL, Patel M. Tetrahedron Lett 1987;28:2499.Boger DL, Patel M. J Org Chem 1988;53:1405.Isochrysohermidin: (e) Boger DL, Baldino CM. J Am Chem Soc 1993;115:11418.Ningalin A, Lamellarin O, Lukianol A, and Storniamide A: (f) Boger DL, Boyce CW, Labroli MA, Sehon CA, Jin Q. J Am Chem Soc 1999;121:54.Ningalin B: (g) Boger DL, Soenen DR, Boyce CW, Hedrick MP, Jin Q. J Org Chem 2000;65:2479. [PubMed: 10789460] Roseophilin: (h) Boger DL, Hong J. J Am Chem Soc 2001;123:8515. [PubMed: 11525659] Ningalin D: (i) Hamasaki A, Zimpleman JM, Hwang I, Boger DL. J Am Chem Soc 2005;127:10767. [PubMed: 16045367] (j) Daly K, Nomak R, Snyder JK. Tetrahedron Lett 1997;38:8611. (k) Joshi U, Josse S, Pipeleir M, Chevallier F, Pradere JP, Hazard R, Legoupy S, Huet F, Dubreuil D. Tetraherdon Lett 2004;45:1031. (1) Muller J, Troschutz R. Synthesis 2006:1513. (m) Naud S, Pipelier M, Chaumette C, Viault G, Adjou A, Huet F, Legoupy S, Aubertin AM, Evain M, Dubreuil D. Eur J Org Chem 2007:3296. (n) Moisan L, Odermatt S, Gombosuren N, Carella A, Rebek J Jr. Eur J Org Chem 2008:1673. (o) Bakkali H, Marie C, Ly A, Thobie-Gautier C, Graton J, Pipelier M, Sengmany S, Leonel E, Medelec JV, Evain M, Dubreuil D. Eur J Org Chem 2008:2156.

13. (a) Boger DL, Baldino CM. J Org Chem 1991;56:6942. (b) Yeung BKS, Boger DL. J Org Chem 2003;68:5249. [PubMed: 12816485]

14. (a) Sakamoto T, Funami N, Kondo Y, Yamanaka H. Heterocycles 1991;32:1387. (b) Heldmann DK, Sauer J. Tetrahedron Lett 1997;38:5791. (c) Sauer J, Heldmann DK, Hetzenegger J, Sichert H, Schuster J. Eur J Org Chem 1998:2885.

15. Streptonigrin: (a) Boger DL, Panek JS. J Am Chem Soc 1985;107:5745.Lavendamycin: (b) Boger DL, Panek JS, Duff SR, Yasuda M. J Org Chem 1985;50:5790.PDE I and II: (c) Boger DL, Coleman RS. J Am Chem Soc 1987;109:2717.CC-1065: (d) Boger DL, Coleman RS. J Am Chem Soc 
1987;110:1321, 4796.Trikentrin A: (e) Boger DL, Zhang M. J Am Chem Soc 1991;113:4230.Pyrimidoblamic Acid: (f) Boger DL, Menezes RF, Honda T. Angew Chem, Int Ed Engl 1993;32:273.P-3A: (g) Boger DL, Honda T, Menezes RF, Colletti SL, Dang Q, Yang W. J Am Chem Soc 1994;116:82.Bleomycin A2: (h) Boger DL, Honda T. J Am Chem Soc 1994;116:5619, 5647.Phomazarin: (i) Boger DL, Hong J, Hikota M, Ishida M. J Am Chem Soc 1999;121:2471.Anhydrolycorinone: (j) Boger DL, Wolkenberg SE. J Org Chem 2000;65:9120. [PubMed: 11149859] Anhydrolycorinone: (k) Boger DL, Wolkenberg SE. J Org Chem 2002;67:7361. [PubMed: 12375965] Minovine: (1) Yuan Q, Ishikawa H, Boger DL. Org Lett 2005;7:741. [PubMed: 15704939] Vindorosine: (m) Elliott GI, Velcicky J, Ishikawa H, Li Y, Boger DL. Angew Chem Int Ed 2006;45:620.Vindoline: (n) Choi Y, Ishikawa H, Velcicky J, Elliott GI, Miller MM, Boger DL. Org Lett 2005;7:4539. [PubMed: 16178578] Ishikawa H, Elliott GI, Velcicky J, Choi Y, Boger DL. J Am Chem Soc 2006;128:10596. [PubMed: 16895428] Vinblastine and vincristine: (o) Ishikawa H, Colby DA, Seto S, Va P, Tam A, Kakei H, Rayl TJ, Hwang I, Boger DL. J Am Chem Soc 2009;131:4904. [PubMed: 19292450] Fendleridine and N-acetylaspidoalbidine (p) J Am Chem Soc 2010;132 in press. 
<smiles>[R]OC(=O)c1[nH]c(C(=O)O)c(-c2c[nH]c3ccccc23)c1-c1c[nH]c2ccccc12</smiles>

1, R = Me, Lycogarubrin C 2, $\mathrm{R}=\mathrm{H}$, Lycogalic Acid (aka Chromopyrrolic Acid) $\operatorname{RebC}(X=O)$
$\operatorname{StaC}\left(X=\mathrm{H}_{2}\right)$<smiles>O=C1NC(O)(C(=O)O)c2c1c1c3ccccc3[nH]c1c1[nH]c3ccccc3c21</smiles><smiles></smiles>

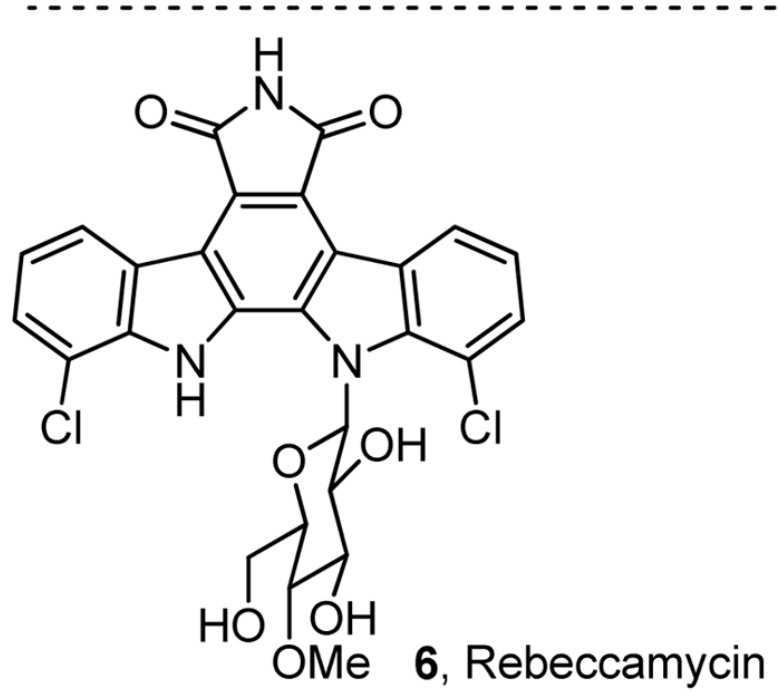

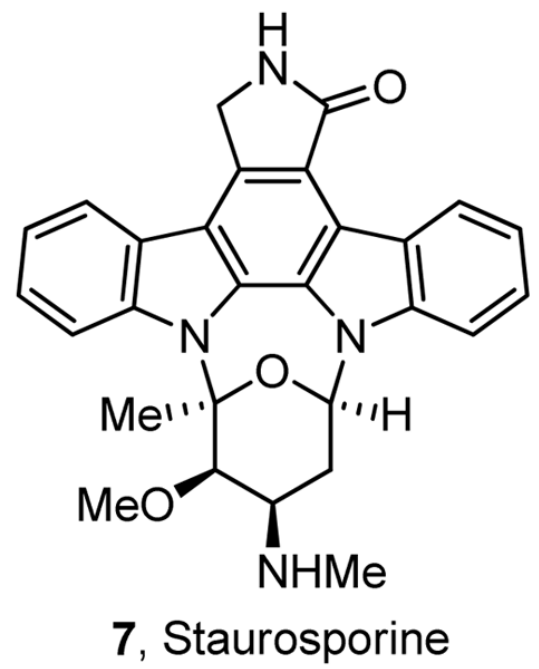

Figure 1.

Natural products. 
<smiles>[R]OC(=O)c1[nH]c(C([R])=O)c(-c2c[nH]c3ccccc23)c1-c1c[nH]c2ccccc12</smiles><smiles>[R]OC(=O)c1nnc(C(=O)OC)c(-c2c[nH]c3ccccc23)c1-c1c[nH]c2ccccc12</smiles><smiles>COC(=O)c1nnc(C(=O)OC)nn1</smiles>

Figure 2.

Initial synthetic strategy. 

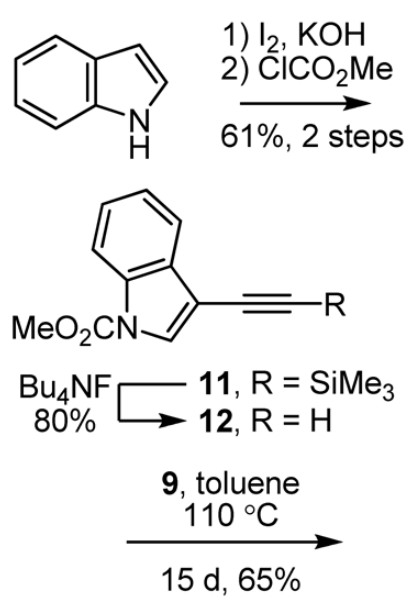

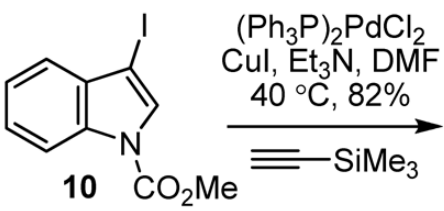

10, $\left(\mathrm{Ph}_{3} \mathrm{P}\right)_{2} \mathrm{PdCl}_{2}$ $\mathrm{Cul}, \mathrm{Et}_{3} \mathrm{~N}, \mathrm{DMF}^{2}$<smiles>[13CH3][13CH3]</smiles><smiles>COC(=O)c1nnc(C(C)=O)c(-c2c[nH]c3ccccc23)c1-c1c[nH]c2ccccc12</smiles>

13

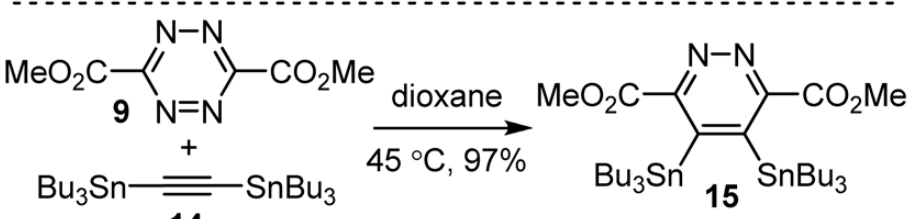

14

10, $\left(\mathrm{Ph}_{3} \mathrm{P}\right)_{2} \mathrm{PdCl}{ }_{2}$ $\mathrm{CuCl}_{2}$. $\mathrm{LiCl}, 60^{\circ} \mathrm{C}$

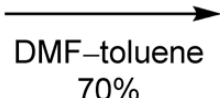<smiles>CC(=O)c1nnc(C(C)=O)c(-c2cn(C(C)=O)c3ccccc23)c1-c1cn(C)c2ccccc12</smiles>

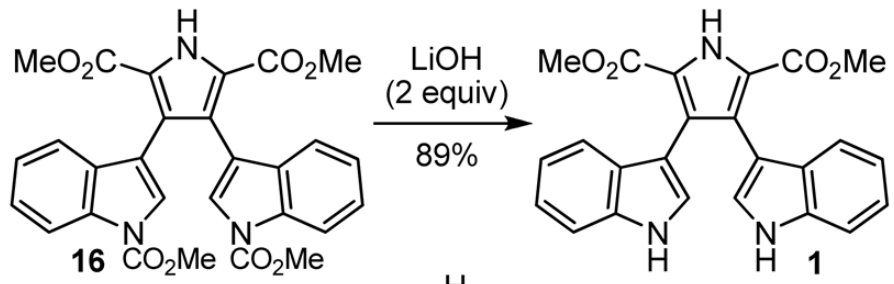<smiles>O=C(O)c1[nH]c(C(=O)O)c(-c2c[nH]c3ccccc23)c1-c1c[nH]c2ccccc12</smiles>

Scheme 1.

Two syntheses of $\mathbf{1}$ and $\mathbf{2}$. 